\title{
Toxoplasma Retinochoroiditis with Chorioretinal Neovascularization in a Young Patient
}

\author{
Nikhila S. Khandwala ${ }^{a}$ Robert A. Hyde ${ }^{b}$ Cagri G. Besirlia \\ ${ }^{a}$ Kellogg Eye Center, University of Michigan, Ann Arbor, MI, USA; bepartment of \\ Ophthalmology and Visual Sciences, University of Illinois, Chicago, IL, USA
}

\section{Keywords}

Choroidal neovascularization - Toxoplasma retinochoroiditis - Subretinal fluid - Retinal lesion

\begin{abstract}
We present a pediatric case to highlight the clinical appearance and management of choroidal neovascularization in the setting of active toxoplasma retinochoroiditis (TRC). A 17-year-old female presented with 2 days of blurry vision in her left eye. Retinal examination demonstrated a pigmented chorioretinal lesion with associated subretinal fluid, vessel sheathing, and adjacent intraretinal hemorrhage. She was diagnosed with active choroidal neovascularization and successful treatment with bevacizumab revealed an underlying active toxoplasmosis lesion. Choroidal neovascularization may rarely present during an acute case of TRC. Dual therapy with anti-vascular endothelial growth factor antibody and anti-parasitic agents leads to improved visual outcomes.
\end{abstract}

\section{Introduction}

Toxoplasma retinochoroiditis (TRC) accounts for a majority of posterior uveitis worldwide $[1,2]$. Toxoplasmosis gondii has an affinity for nervous tissue, specifically the retinal ganglion cells in the eye [3]. TRC occurs in healthy children or young adults in $75 \%$ of cases, often during puberty [1,4]. Various clinical presentations have been reported, including destructive active retinitis with associated vitritis [4]. Less commonly, retinal punctate lesions with subretinal exudate and minimal vitreous reaction occur in up to $3 \%$ of patients [4]. As a progressive and recurring necrotizing retinitis, TRC is associated with long-term visionthreatening complications including retinal detachment, glaucoma, and choroidal neovascular membrane (CNVM) [2]. While CNVM is a well-known late complication of TRC, there are 
few reports of CNVM during periods of acute infection and inflammation [5, 6]. We report the clinical presentation and management of a patient presenting with choroidal neovascularization which initially masked active TRC and was successfully treated with dual anti-VEGF and anti-parasitic therapy.

\section{Case Report}

A 17-year-old previously healthy immunocompetent female presented with sudden onset distortion in vision of her left eye that she described as a "bicycle spoke" for 2 days. Her best-corrected visual acuity (BCVA) at presentation was 20/60 in the left eye with a superior nasal deficiency on confrontational visual fields. Anterior segment examination of the left eye was remarkable for $1+$ cell in the anterior chamber. The BCVA for the right eye was $20 / 20$, and the anterior and posterior segment exam was unremarkable. Fundus exam of the left eye demonstrated an inferotemporal pigmented chorioretinal lesion. There was a large area of subretinal fluid associated with the pigmented chorioretinal lesion. Subtle sheathing of the inferotemporal arterioles and venules and adjacent intraretinal hemorrhage were noted on biomicroscopic evaluation (Fig. 1a). Fluorescein angiography of the left eye demonstrated blocking early in the area of the pigmented chorioretinal lesion with late leakage and pooling (Fig. 1b, c). Optical coherence tomography (OCT) showed a focal area of retinal thinning inferotemporally with adjacent subretinal lesion and a large amount of subretinal fluid (Fig. 1d). Macular OCT demonstrated subfoveal tracking of fluid from inferotemporal macula and cystic intraretinal fluid centrally (Fig. 1e). Vitreous opacities
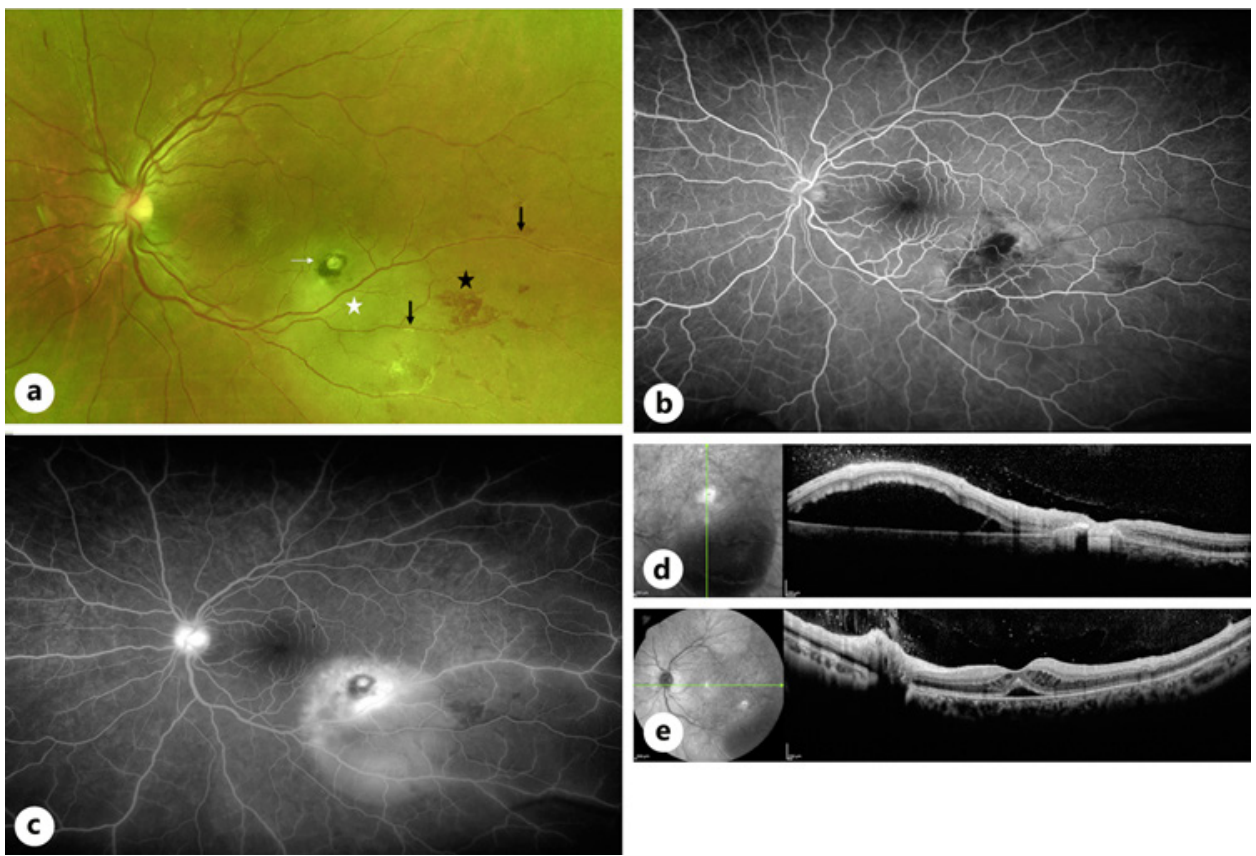

Fig. 1. a Color fundus photograph demonstrating inferotemporal pigmented chorioretinal lesion (white arrow) with associated subretinal fluid (white star). Sheathing of the inferotemporal arterioles and venules (black arrows) and adjacent intraretinal hemorrhage (black star). b, c Fluorescein angiography demonstrating early blocking in the area of the pigmented chorioretinal lesion with late leakage and pooling. $\mathbf{d}$ OCT demonstrating a focal area of retinal thinning with adjacent subretinal lesion and a large amount of subretinal fluid. e Macular scan demonstrating subfoveal fluid. OCT, optical coherence tomography. 
were noted overlying the area of inferotemporal subretinal fluid as well as the optic nerve (Fig. 1d, e).

The clinical and imaging findings were consistent with an active CNVM, and the patient was treated with an intravitreal injection of bevacizumab $(1.25 \mathrm{mg} / 0.05 \mathrm{~mL})$. She presented for follow-up 2 weeks after receiving the injection with floaters although vision was subjectively improved. Fundus examination 5 weeks after initial presentation demonstrated near complete resolution of subretinal fluid, no vitreous cell, and active retinochoroiditis adjacent to the old toxoplasmosis scar (Fig. 2a, b). Macular scan demonstrated resolution of subfoveal fluid (Fig. 2c). The rapid resolution of subretinal and intraretinal fluid following intravitreal anti-VEGF therapy confirmed the diagnosis of CNVM while revealing the TRC lesion. Given the concern for active TRC, she was treated with oral trimethoprim-sulfamethoxazole (800-160 $\mathrm{mg}$ ) monotherapy daily for a period of 5 months and did not require additional bevacizumab injections. The patient was followed-up for a period of 5 months, with notable improvement in inflammation and a final BCVA of $20 / 20$.

\section{Discussion}

Choroidal neovascularization associated with TRC was first described in 1969 by Friedmann and Knox [7] as a late complication of this parasitic infection. It has since been recognized as one of the most common late complications of TRC, occurring in up to 19\% of patients within 26 years after initial diagnosis [8, 9]. There are few case reports, however, of CNVM during the acute infectious phase of TRC. Willerson et al. [10] described the first case of subretinal neovascularization in association with active TRC in 1977. A case report by Hegde et al. [5] cited 3 cases of coexisting CNVM with active TRC. All patients presented with subretinal hemorrhage adjacent to the pigmented lesion. One patient presented in their teenage years with history of bilateral ocular toxoplasmosis and unilateral CNVM with active TRC on presentation. The patients were treated with a combination of anti-VEGF, trimethoprim/sulfamethoxazole , and oral corticosteroids, with resolution of the retinitis within 6 weeks and CNVM within 6-12 weeks [5]. Rishi et al. [11] presented another example of CNVM secondary to TRC in a teenager. The patient presented with history of bilateral ocular toxoplasmosis, with unilateral subfoveal CNVM and subretinal fluid adjacent to active TRC. The patient was managed with a combination of photodynamic therapy and
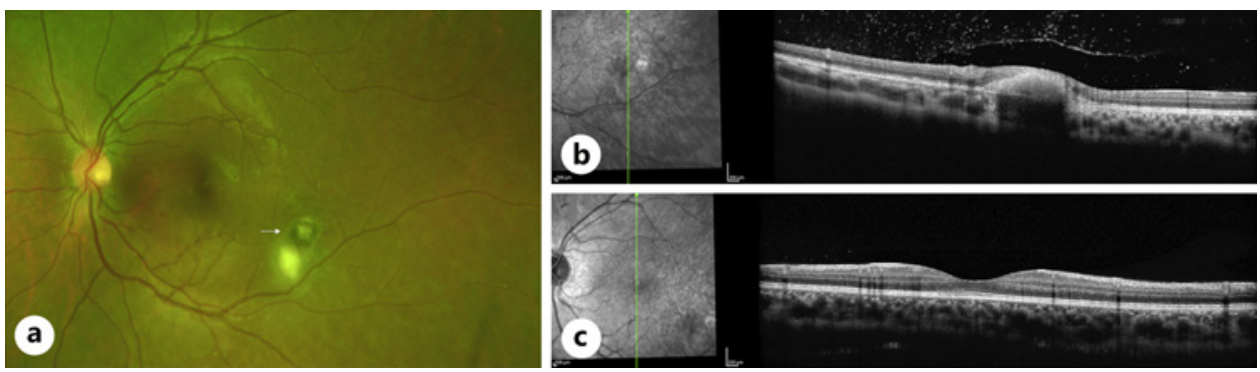

Fig. 2. a Color fundus photograph 5 weeks after initial presentation demonstrating inferotemporal pigmented chorioretinal scar (white arrow) with improvement of subretinal fluid revealing active retinochoroiditis adjacent to the old toxoplasmosis scar. b OCT demonstrating active TRC lesion with overlying vitreous cell. c Macular scan demonstrating resolution of subfoveal fluid. *Image copyright holder is the Department of Ophthalmology and Visual Sciences at the University of Michigan W.K. Kellogg Eye Center. OCT, optical coherence tomography. 
intravitreal anti-VEGF injection after a period of 2 days, with resolution of CNVM within 8 weeks [11].

While late-onset CNVM presents adjacent to retinochoroidal scars in healed TRC, CNVM alongside active TRC may be seen anywhere within the active retinitis lesion [5, 11]. CNVM with active TRC seems to occur more commonly in younger patients with acquired toxoplasmosis, and may lead to vision loss, especially in cases of foveal involvement [11]. CNVM with active TRC typically presents with minimal vitritis, a yellow-white necrotizing retinitis lesion with adjacent subretinal hemorrhage, macular thickening, and subretinal fluid [5]. In contrast to the above case reports, our patient presented in adolescence with a unilateral pigmented chorioretinal lesion adjacent to CNVM with overlying vitritis and subretinal fluid. The extramacular location of TRC may have contributed to the delayed presentation of our patient, resulting in the concomitant presentation of a CNVM with significant activity.

The pathogenesis of CNVM associated with late TRC is thought to be attributed to the preferential selection of retinal microvascular endothelium by T. gondii tachyzoites [12]. By contrast, during the active phase of TRC, it has been postulated that ongoing retinitis impedes venous outflow leading to active vasoproliferation $[5,6]$. It is hypothesized that some patients develop CNVM during active TRC due to the intense retinal inflammation causing a break in Bruch's membrane and choriocapillaris [5].

It can be difficult to distinguish active infectious retinitis in the setting of a CNVM, as demonstrated in this case. The lack of vitritis on clinical examination and masking of active retinochoroiditis by the large amount of subretinal fluid in our patient concealed the underlying TRC. However, the acute infectious retinitis was revealed by the successful treatment of CNVM and resolution of subretinal fluid and implementation of anti-parasitic therapy led to a quick response and improvement of vision.

\section{Conclusion}

We present a rare pediatric case of choroidal neovascularization in the setting of active TRC. Choroidal neovascularization rarely presents in the setting of active TRC, especially in the pediatric population. A combination of anti-vascular endothelial growth factor antibody and anti-parasitic agents leads to significant improvement in visual acuity.

\section{Statement of Ethics}

Written informed consent for patient information and images to be published was provided by the patient.

\section{Conflict of Interest Statement}

The authors have no conflicts of interest to declare.

\section{Funding Sources}

The authors received no financial support for the research, authorship, and/or publication of this article. 
Khandwala et al.: Choroidal Neovascularization with Active Toxoplasmosis

\section{Author Contributions}

C.G.B. was responsible for the conception and design of this project. N.S.K. and R.A.H. were responsible for data collection, data analysis, and interpretation. N.S.K. was responsible for drafting of the manuscript. R.A.H. and C.G.B. were responsible for critical revision of the article. Final approval of the version to be submitted for publication was performed by N.S.K., R.A.H., and C.G.B.

\section{References}

1 Smith JR, Cunningham ET. Atypical presentations of ocular toxoplasmosis. Curr Opin Ophthalmol. 2002 Dec; 13(6):387-92.

2 Park YH, Nam HW. Clinical features and treatment of ocular toxoplasmosis. Korean J Parasitol. 2013 Aug; 51(4):393-9.

3 Noble KG, Carr RE. Toxoplasma retinochoroiditis. Ophthalmology. 1982 Nov;89(11):1289-90.

4 Cotliar AM, Friedman AH. Subretinal neovascularisation in ocular toxoplasmosis. Br J Ophthalmol. 1982 Aug; 66(8):524-9.

5 Hegde S, Relhan N, Pathengay A, Bawdekar A, Choudhury H, Jindal A, et al. Coexisting choroidal neovascularization and active retinochoroiditis-an uncommon presentation of ocular toxoplasmosis. J Ophthalmic Inflamm Infect. 2015;5:22.

6 Mushtaq F, Ahmad A, Qambar F, Ahmad A, Zehra N. Primary acquired toxoplasma retinochoroiditis: choroidal neovascular membrane as an early complication. Cureus. 2019 Feb 4;11(2):e4001.

7 Friedmann CT, Knox DL. Variations in recurrent active toxoplasmic retinochoroiditis. Arch Ophthalmol. 1969 Apr;81(4):481-93.

8 Atmaca LS, Simsek T, Batioglu F. Clinical features and prognosis in ocular toxoplasmosis. Jpn J Ophthalmol. 2004 Aug; 48(4):386-91.

9 Skorska I, Soubrane G, Coscas G. [Toxoplasmic choroiditis and subretinal neovessels]. J Fr Ophtalmol. 1984; $7(3): 211-8$.

10 Willerson D, Aaberg TM, Reeser F, Meredith TA. Unusual ocular presentation of acute toxoplasmosis. Br J Ophthalmol. 1977 Nov;61(11):693-8.

11 Rishi P, Venkataraman A, Rishi E. Combination photodynamic therapy and bevacizumab for choroidal neovascularization associated with toxoplasmosis. Indian J Ophthalmol. 2011 Feb;59(1):62-4.

12 Smith JR, Franc DT, Carter NS, Zamora D, Planck SR, Rosenbaum JT. Susceptibility of retinal vascular endothelium to infection with Toxoplasma gondii tachyzoites. Invest Ophthalmol Vis Sci. 2004 Apr;45(4):1157-61.

\section{Karger'}

